\title{
Feedback Control in an Actuated Acoustic Guitar Using Frequency Shifting
}

\author{
ETIENNE THUILLIER', OTSO LÄHDEOJA ${ }^{2}$, AND VESA VÄLIMÄKI, ${ }^{1}$ AES Fellow \\ (etienne.thuillier@aalto.fi) (otso.lahdeoja@uniarts.fi) (vesa.valimaki@aalto.fi) \\ ${ }^{1}$ Aalto University, Acoustics Lab, Department of Signal Processing and Acoustics, FI-02150 Espoo, Finland \\ ${ }^{2}$ University of the Arts Helsinki, Center for Music \& Technology, Helsinki, Finland
}

\begin{abstract}
Recent work has demonstrated that the classical guitar can be advantageously augmented using a pickup to drive an actuator mounted on the guitar's back plate, thereby allowing enrichment of the instrument's timbral palette with audio effect processors in the loop. The feedback problem that results from such setup is similar to that occurring in live music performance setups where the sound of a guitar is amplified using a loudspeaker. In the case at hand measurements of the augmented guitar's open loop response demonstrate that instabilities are susceptible to first occur from the string's modes and not from the guitar's soundbox. In particular, the shape of the magnitude response suggests frequency shifting as a viable solution to string instability. Introduction of an upwards frequency shift in the forward path is proposed as a means for stabilizing the closed loop system. Experimental results demonstrate that the proposed solution leads to improved stability even for a modest frequency shift of $3 \mathrm{~Hz}$. The achieved gain margin improvement, which is shown to be of at least $3 \mathrm{~dB}$, then comes at the cost of a clearly perceptible amplitude modulation, which may be acceptable in conjunction with other audio effects chosen by the performer.
\end{abstract}

\section{INTRODUCTION}

Acoustic feedback occurs commonly in public address and live music sound systems, when the sound picked up by a microphone is amplified and played through a loudspeaker located in close vicinity to the microphone. A loud howling sound is then often generated. An extensive body of research work addresses this problem, an exhaustive review of which is provided by van Watershoot and Moonen [1]. According to the classification operated in this review, acoustic feedback control techniques can be broadly distinguished according to the way in which they attempt to prevent the occurrence of instability. Specifically, phase-modulating feedback control (PFC) methods $[2,3,1]$ smoothen the magnitude of the system's open loop response, notch-filterbased howling suppression (NHS) methods suppress the howling effect, and adaptive feedback cancellation (AFC) methods decouple the loudspeaker from the microphone by way of estimating the channel.

Frequency shifting (FS), is a special case of the PFC method originally introduced by Schroeder as a solution to the problem of acoustic feedback in public address systems [4-7]. In contrast to other PFC methods, FS generates a single sideband while providing complete carrier suppression [3], which makes it a simple case to interpret technically.
A theoretical added gain margin from 4 to $10 \mathrm{~dB}$ was derived in the case of single-channel public address systems following statistical properties of the magnitude response of the loudspeaker-to-microphone transmission channel in large rooms [7-9]. This theoretical foundation was later extended by Poletti, with applications to multichannel systems [10], and by Zheng [11] to cases in which the direct transmission path dominates and cannot be neglected. Early on, a detrimental amplitude modulation artifact was identified as a limit to the applicability of the method [7].

Feedback is employed in the field of augmented instruments where it is proposed as a means for augmenting traditional instruments $[12,13]$. In the case of the present study, a nylon-stringed classical guitar is augmented with a pickup, a processing, and actuation system comprising a piezoelectric under-saddle pickup, outboard audio effects processing on a $\mathrm{PC}$, an audio amplifier, and a structureborne sound driver mounted on the guitar's back plate. The guitar's design is detailed in a previous publication [14]. The rationale of the setup is to provide a means to utilize electronic sounds in a classical guitar performance, radiating directly from the instrument itself. These sounds can be external to the instrument (sample playback), or processed from the guitar's signal. In the latter case, a closed signal loop occurs between the pickup and the transducer 
through the instrument's soundbox and strings, giving rise to instabilities at the string or soundbox's modes.

The soundbox instability aspect of the problem is particularly undesirable. A similar problem occurs in the case of live amplification of acoustic guitar performances. The sound propagates back towards the guitar's top plate, which can then enter in resonance to suddenly produce a disturbing feedback sound. Occluding the sound hole of the guitar partially mitigates the feedback problem by way of dampening the top plate, but this results in a significant alteration of the instrument's sound [15]. Recently, active control of the top plate's modes in real time has been proposed $[16,17]$.

Regarding the string instability aspect, feedback can potentially bring extended sustain or swelling of the string's vibration. While the extended sustain effect is a desirable feature of the system under study, the swelling of vibration is detrimental to the instrumentalist's performance as it builds up uncontrollably into loud narrowband sounds if the offending string is not muted by the player.

The above stability problems are investigated under the formalism of control theory. In particular, the conditions for stability are interpreted from the system's open loop response in light of the Nyquist criterion. In the case at hand, the transmission path from the actuator towards the backplate, front-plate, bridge, and strings of the instrument is unknown. In particular, the above-mentioned theoretical added gain margin improvement when applying FS in the case of public address systems is not transposable owing to differences in transmission channel from the actuator to the pickup.

Correspondingly, a first contribution of this paper lies in the measurement of the specific open loop response of the actuated guitar setup. In particular, sharp resonance peaks at the string's modal frequencies are shown to exceed the contribution from the soundbox throughout the frequency range. This result provides evidence that instabilities are susceptible to first occur from the string's modes and not from the soundbox, which justifies prioritizing the string instability aspect of the problem over that of the soundbox. In particular, the shape of the magnitude response suggests FS as a viable solution to string instability.

A second contribution of this work lies in the application of FS in the forward loop as a means for improving the gain margin before instability of the system. Experiments demonstrate that a modest frequency shift of $3 \mathrm{~Hz}$ provides an improvement of at least $3 \mathrm{~dB}$ SPL before instability. The resulting amplitude modulation artifact lies under the 4-6 Hz modulation range of the vibrato technique [18]. The amplitude modulation aspect of the vibrato technique is indeed known as its most salient perceptual feature, even more so than pitch modulation [19]. Therefore, a persistent vibrato effect is avoided to the benefit of more subtle amplitude fluctuation artifacts, which ensures wider musical application of the method.

This paper is organized as follows. Sec. 1 presents a linear model of the actuated guitar setup and the conditions for stability under such a linear assumption. Sec. 2 proposes the application of FS as a solution to the problem under study.
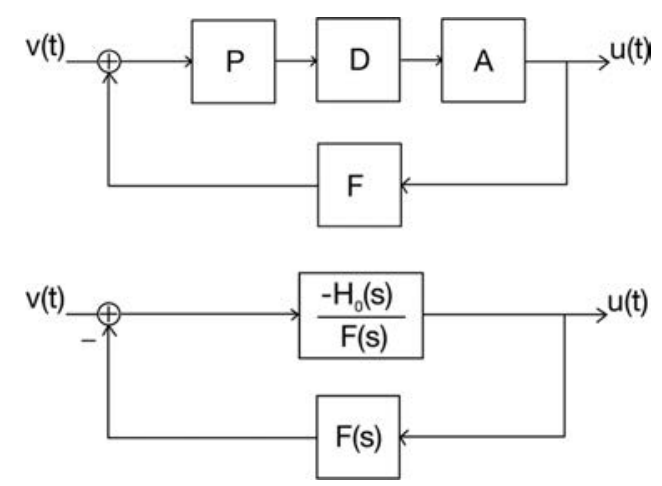

Fig. 1. Block diagram of the closed loop system formed by the actuated guitar setup top. Rearrangement of the block diagram (bottom) under the linear assumption and according to Eq. (2).

Sec. 3 reports the experimental methodology and setup carried out for the system's open loop measurements and the evaluation of FS as a candidate solution. Sec. 4 reports and discusses the corresponding results. Sec. 5 concludes this paper.

\section{LINEAR MODEL}

This section presents a linear model of the actuated guitar setup and the conditions for stability under such a linear assumption.

\subsection{Feedback Loop}

Borrowing from the terminology used in the classical problem of acoustic feedback control for public address systems [1], the actuated guitar setup described above comprises a feedback loop formed by an electroacoustic forward path (potentially nonlinear) and a passive feedback path (assumed linear). In the forward path, the piezoelectric saddle pickup senses vibrations from the string and the resulting audio signal is transformed, amplified, and fed into the contact actuator. In the feedback path, the vibrations induced (by the actuator) at the back plate propagate back towards the saddle pickup and the guitar strings through mechanical and acoustical couplings.

A model of the feedback loop of the actuated guitar setup is given in the block diagram of Fig. 1 (top), in which:

- $v(t)$ models the pickup signal resulting from a string plucking force, as function of time $t$;

- $P$ models the piezoelectric saddle pickup;

- $D$ models, in continuous time, a signal processing platform including an analog-to-digital converter, digital audio amplification, digital audio effects, and digital-to-analog converter;

- A models analog amplification, the actuator, and its coupling to the back plate of the guitar;

- $F$ models the feedback path from back plate to the guitar's pickup;

- $u(t)$ models the force signal exerted on the back plate. 
Assuming the components of Fig. 1 (top) are linear and time-invariant, the closed loop transfer function of the system can be written as

$$
\begin{aligned}
H(s) & =\frac{U(s)}{V(s)}, \\
& =\frac{P(s) D(s) A(s)}{1-P(s) D(s) A(s) F(s)} .
\end{aligned}
$$

where $U$ and $V$ denote the Laplace transforms of signals $u$ and $v$, respectively, and $s$ denotes the Laplace variable, which is complex-valued.

\subsection{Nyquist Criterion}

The problem introduced above can be expressed as a control problem. Specifically, Eq. (1) can be re-formulated as:

$$
H(s)=\frac{-H_{0}(s) / F(s)}{1+H_{0}(s)},
$$

where

$$
H_{0}(s)=-P(s) D(s) A(s) F(s)
$$

denotes the open loop response of the system following conventional notation in the field of control theory, in which feedback networks are drawn with a negative feedback junction as in Fig. 1 (bottom).

Specifically, we seek to scale the magnitude response of $H$ upwards by acting on $D$ without causing instability. Such stability requirement is met when none of the poles of $H$ lies rightwards of the imaginary axis on the complex $s$ plane. The Nyquist criterion provides a practical method to study this condition from the open loop response Eq. (3), including gain or phase margins before instability the case being [20].

The open loop system is stable given the definitions provided above for the network's components. Equivalently, none of its poles lies rightwards of the imaginary axis. In this case, the Nyquist criterion implies that the number of poles of the closed loop system $H$ lying on the right-hand side of the complex plane is directly given by the number of (clockwise) encirclements of point -1 in the complex plane by the open loop system's Nyquist plot [20, 21]. Instability thus occurs when at least one encirclement is observed, or equivalently, if at least one point of the Nyquist plot lies on the real axis leftwards of the -1 value [1]:

$$
\left\{\begin{array}{l}
\left|H_{0}(\mathrm{i} 2 \pi f)\right| \geq 1, \\
\angle H_{0}(\mathrm{i} 2 \pi f)=n \pi, \quad n \in \mathbb{Z},
\end{array}\right.
$$

where $\mathrm{i}$ is the imaginary unit and $f$ denotes frequency in $\mathrm{Hz}$.

\section{FREQUENCY SHIFTING}

This section discusses the application of FS as a feedback control method to the problem under study.

A frequency-shifted discrete-time signal $y(n)$ can be derived from the sampled pickup signal $x(n)$ according to $[22,23]$ :

$$
y(n)=2 \Re\left\{[x(n) * h(n)] \mathrm{e}^{\mathrm{i} 2 \pi f_{\text {shift }} n \mathrm{~T}_{\mathrm{s}}}\right\},
$$

where $n$ denotes the sample index, $\Re$ the real part, * the discrete-time convolution operator, $T_{\mathrm{s}}=1 / F_{\mathrm{s}}$ the sampling interval (inverse of sample rate), $f_{\text {shift }}$ the frequency shift offset in $\mathrm{Hz}$ and $h(n)$ is the impulse response of a complex half-band filter approximating the following ideal frequency response [24]:

$$
H_{\text {ideal }}\left(\mathrm{e}^{\mathrm{i} \omega}\right)=\left\{\begin{array}{cc}
1 & 0<\omega<\pi \\
0 & -\pi<\omega<0 .
\end{array}\right.
$$

The above frequency response is that of an ideal halfband lowpass filter after a rotation of $\pi / 2$ radians. An implementation of the complex half-band filter can thus be achieved from a half-band low-pass filter prototype $g(n)$ according to [24]:

$$
h(n)=\mathrm{i}^{n} g(n),
$$

where $\mathrm{i}^{n}$ carries out the above mentioned $\pi / 2$ rotation in time domain.

When a positive-valued frequency shift is introduced in the feedback loop, frequency components are shifted further to the right in the spectrum with each excursion of the signal around the loop. With each frequency shift, the component is affected by a gain factor that corresponds to the open loop's magnitude response value at the shifted position. The gain level imparted on an input frequency component $f_{0}$ after $C$ cycles in the loop is thus given by [7]:

$$
\begin{aligned}
L\left(C, f_{0}\right) & =\sum_{c=0}^{C-1} l\left(f_{0}+c f_{\text {shift }}\right), \\
& \approx C \bar{l} \quad \text { for } C \gg 1,
\end{aligned}
$$

where $l(f)$ denotes the open-loop gain in $\mathrm{dB}$, i.e. $l(f)=$ $20 \log _{10}\left|H_{0}(\mathrm{i} 2 \pi f)\right|$, and $\bar{l}$ denotes the average open-loop gain.

As $C$ tends to infinity, the above expression converges and stability is ensured when the average level of the open loop gain lies under $0 \mathrm{~dB}$ [7]:

$$
\bar{l}<0 \text {. }
$$

Under this condition, FS provides a guarantee of stability for systems with open loop responses reaching beyond the 0 - $\mathrm{dB}$ threshold locally, provided the magnitude of the response lies under $0 \mathrm{~dB}$ globally. Experimental measurements of the system's open loop response are provided in this work to determine the applicability of FS as a candidate solution for the soundbox instability and string instability problems. In particular, the open loop response is conducted both in muted string and non-muted string configurations. The former allows evaluating FS as a candidate to the soundbox instability problem, while the later allows evaluating FS as a candidate to the string instability problem.

\section{EXPERIMENTAL SETUP}

In this experimental study, a classical guitar from Perez was fitted with a Fishman classic 4 preamplifier and piezoelectric saddle pickup. A Tectonic Elements TEAX32C304/B actuator was fastened to the back plate using industrial adhesive. The exact positioning of the actuator is pictured 

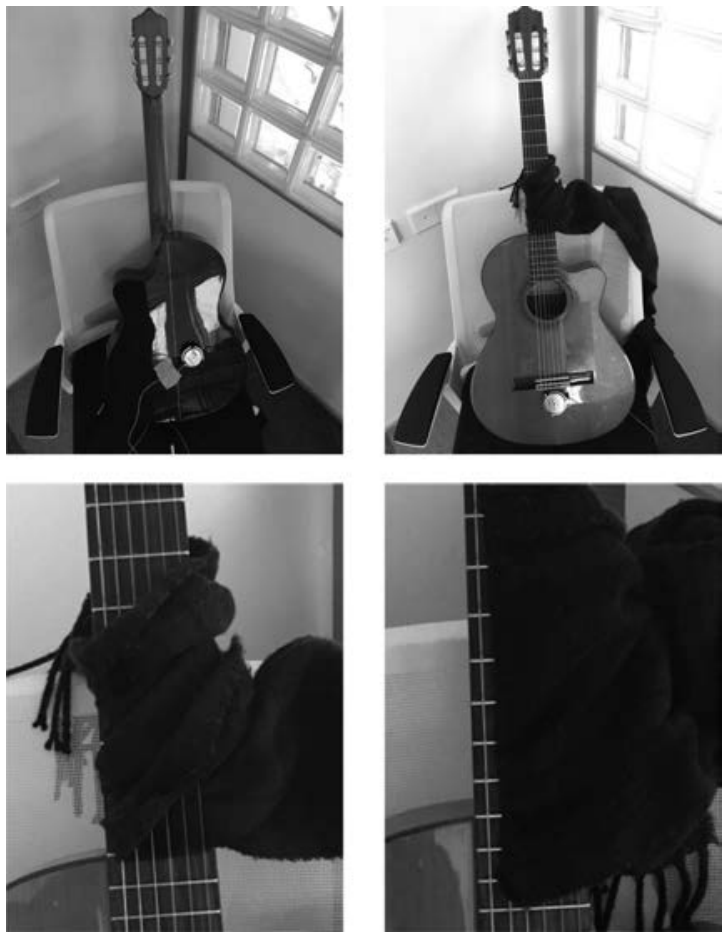

Fig. 2. Experimental setup of the actuated guitar. Positioning of back plate actuator (top left). Muted string configuration for open loop response measurement (top right and bottom left). Open E2 configuration for open loop response measurement (bottom right). The front plate actuator was not used in this work.

in Fig. 2 (top left) and was determined in collaboration with Uwe Florath, Master Luthier in Helsinki, by trial and error, in order to optimize the spectral balance of the sound output. An asymmetrical position has been sought in order to minimize standing waves on the back plate. The sound delivered by the saddle pickup was captured in real time using a MOTU UltraLite mk3 USB sound card set to a sample rate of $48 \mathrm{kHz}$ and an input-output buffer size of 64 samples. The sine-sweep procedure, digital gain, and frequency shifting were implemented using a VST plug-in instantiated in a Max MSP patch. The actuator signal was amplified using a 240-W audio amplifier.

\subsection{Open Loop Response Estimation}

A measurement of the system's open loop response (without FS) was conducted with all strings muted as well as in an open E2 string configuration, in which all but the lowest string of the guitar were muted using a cloth. Photographs of these configurations are provided in Fig. 2 (top left and top right). The guitar was positioned to rest vertically on an office chair and was not moved between sine sweep measurements. The open loop frequency responses were measured under an identical loop gain setup for both configurations. In practice, the loop gain was set by trial and error so as to produce a slow build up of the freelyringing string in open E2 configuration, starting from a quiescent state after the system was switched in closed loop mode.
The open loop frequency response estimate is given by:

$$
\hat{H}_{0}(\mathrm{i} \omega)=-\frac{M(\mathrm{i} \omega)}{E(\mathrm{i} \omega)},
$$

where $M(i \omega)$ denotes the measured signal provided by the guitar pickup in response to a probe signal $E(\mathrm{i} \omega)$ generated by the signal processing platform $D$ and fed into the system in open loop state. In practice, an exponential sine sweep method was implemented $[25,26]$. This allows to extract the linear contribution of the response and isolate it from eventual mild nonlinearities [27], such as that of the actuator and its coupling to the guitar.

\subsection{Measurements with Frequency Shifting}

A quantitative evaluation of the FS method was conducted as described above using the open E2 configuration of Fig. 2 (bottom right), but in this case with the instrument held by a guitar player sitting in playing stance. Specifically, a plucking force of $10 \mathrm{~N}$ was applied on the low $\mathrm{E}$ string, perpendicularly from the string in a plane parallel to the top plate. This was realized accurately by way of pulling a thin cotton thread tied to the guitar string approximately $4.5 \mathrm{~cm}$ from the bridge. A force meter was used to precisely set the pulling force exerted on the string at which point the thread was burned with a lighter to release the string in a simulated plucking action, following the method taught in [28].

The plucking experiment was carried out with and without FS in the forward path for several loop gain setups. For each experimental condition, the plucking experiment was repeated three times. Each time, the sound radiated by the instrument was captured by a dynamic microphone (Sennheiser e822) placed at a distance of $20 \mathrm{~cm}$ in front of the guitar sound hole and recorded. The peak sound pressure level (SPL) was also taken during one plucking experiment using an A-weighted SPL meter. This reading was compared against the maximum of the A-weighted envelope of the corresponding recording so as to derive a calibration offset. In practice, the average A-weighted envelope was computed from the recordings of each experimental condition and adjusted according to said offset to produce the final results.

In particular, the final result comprises SPL curves produced for a reference loop gain level corresponding to the threshold of near instability for the unmodified augmented guitar setup, i.e., MSG without FS. SPL curves were produced with FS from that gain level upwards with a frequency shift in the forward path. In practice, the low-pass half-band prototype filter $g(n)$ of Eq. (7) was implemented as an $8^{\text {th }}$ order minimum-phase IIR filter so as to minimize the incurred latency. Specifically, an elliptic filter design was used to provide a sharp transition band [24]. The specification for the complex half-band filter is provided in Table 1, along with the corresponding low-pass filter prototype design parameters. The latency incurred by the complex half-band filter lies under $1 \mathrm{~ms}$ almost throughout the auditory band with a maximum of $10 \mathrm{~ms}$ reached in the vicinity of the filter's transition bands. 
Table 1. Cut-off frequency and stop-band ripple specifications of the complex half-band filter $g(n)$ and low-pass filter $h(n)$.

\begin{tabular}{llccc}
\hline & $f_{\text {pass }}$ & $\delta_{\text {pass }}$ & $f_{\text {stop }}$ & $\delta_{\text {stop }}$ \\
\hline$g(n)$ & $\frac{f_{s}}{4}-82.4$ & $6 \mathrm{~dB}$ & $\frac{f_{s}}{4}$ & $40 \mathrm{~dB}$ \\
$h(n)$ & $82.4 \mathrm{~Hz}$ & $6 \mathrm{~dB}$ & $0 \mathrm{~Hz}$ & $40 \mathrm{~dB}$ \\
\hline
\end{tabular}

\section{RESULTS}

This section presents and discusses the experimental results obtained following the methodology described in the previous section.

\subsection{Open Loop Response Measurements}

As shown in Fig. 3, the measured open loop magnitude response in muted string configuration and under the specified loop gain setup, lies about $3 \mathrm{~dB}$ under the magnitude threshold of Eq. (4) throughout the audio range. Harmonic distortion for the first four distortion terms was found to lie under $7 \%$ in the range from $50 \mathrm{~Hz}$ to $450 \mathrm{~Hz}$. This lowfrequency end dominates the response with three broad maxima located around 73, 122, and $222 \mathrm{~Hz}$ respectively. Body modes stability is ensured for increased loop amplification within the above mentioned gain margin of $3 \mathrm{~dB}$, assuming the guitar is maintained in the specified measurement position.

For higher levels of loop amplification, stability could be ensured with proper conditioning of the phase response to avoid meeting both conditions of Eq. (4) at any given frequency. The open loop phase response is given for the low frequency end in Fig. 4 (middle). Unfortunately, the phase wraps several times around the $2 \pi$ mark, causing the instability condition of Eq. (4) to be met at each of these $2 \pi$ rotations. This results from the propagation time of the signal across the open loop system. In particular, the signal processing chain introduces a delay (latency) of slightly more than $8 \mathrm{~ms}$, as indicated by a dashed line in the open loop impulse response of Fig. 4 (top). However, the propagation time across the soundbox is also significant at low frequencies, as indicated by the slope of the phase response of Fig. 4 (bottom) in which the digital delay has been compensated. It is unclear how the effect of such delays on the phase term could be compensated.

For the same loop gain level, Fig. 5 (top) shows that coupling to the open E2 string introduces harmonic resonances with crossings of the magnitude threshold Eq. (4). As ex-

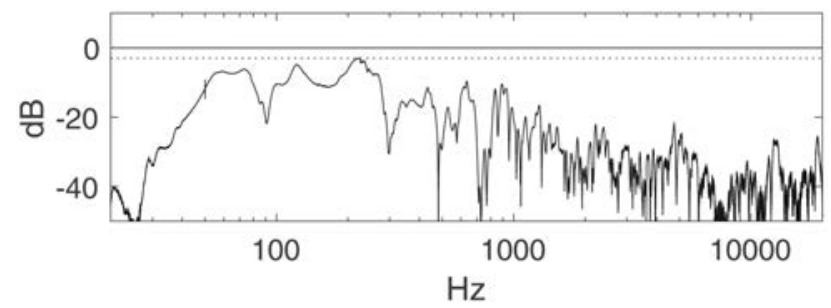

Fig. 3. Open loop magnitude response in muted strings configuration. The $-3 \mathrm{~dB}$ mark is indicated by the dotted line.
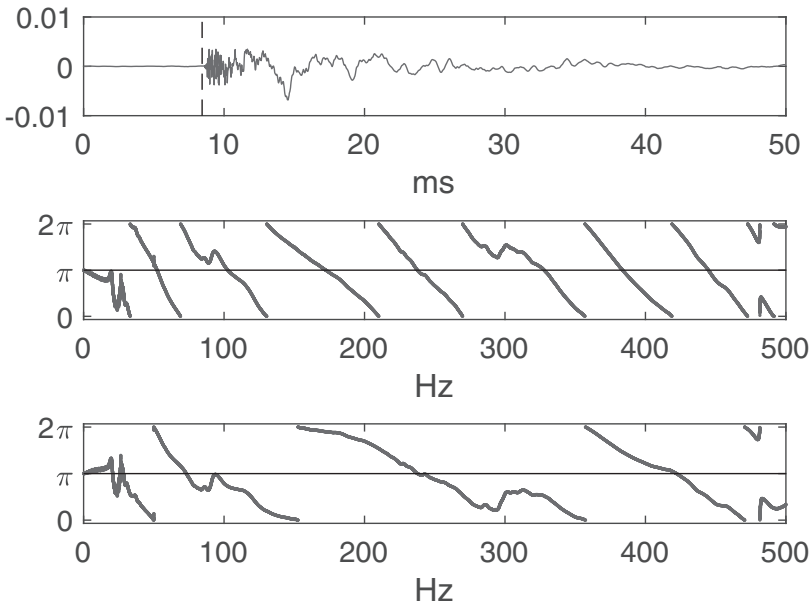

Fig. 4. Open loop impulse response in muted strings configuration (top), with the platform latency of about $8 \mathrm{~ms}$ marked with the dashed line, the corresponding low-frequency phase response with the platform delay (middle) and without it (bottom).
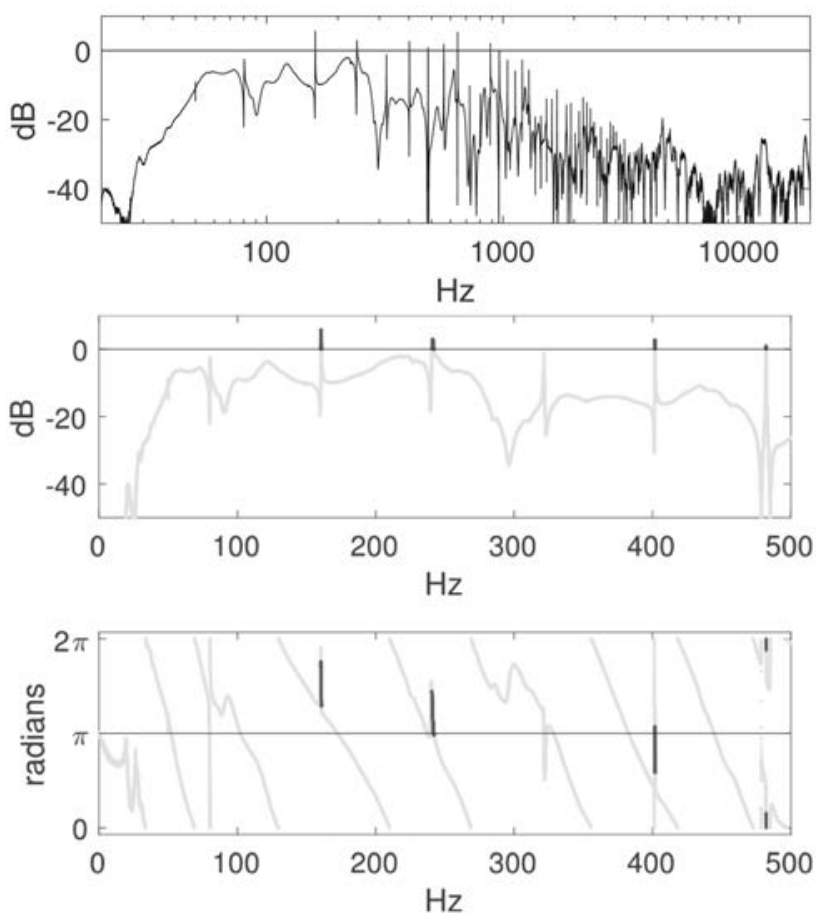

Fig. 5. Open loop magnitude response in open E2 configuration (top). Low end magnitude (middle) and phase (bottom) responses with values marked in black at frequencies meeting the magnitude condition of instability in Eq. (4).

pected, the string resonances are sharp exhibiting a high quality factor. Consequently, the excursion of the phase response around the resonance frequencies is important, as pictured in Fig. 5 (bottom). This leads to a high probability for the phase condition of Eq. (4) to be met despite the relatively narrow bands of the resonance above the magnitude condition, as pictured in Fig. 5 (middle). In the measured case, both conditions for instability Eq. (4) are met around $400 \mathrm{~Hz}$, which corresponds to the fifth harmonic of the 

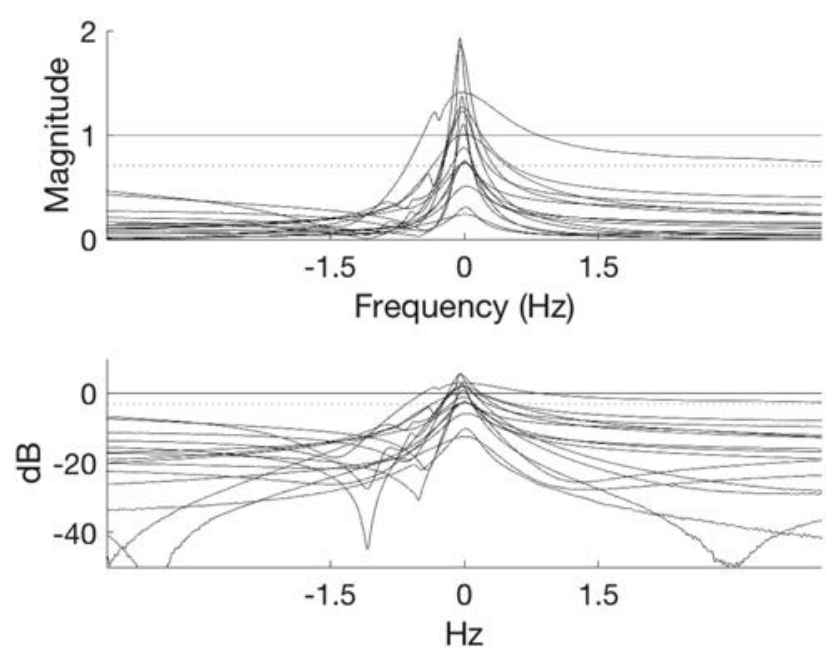

Fig. 6. Centered peak magnitudes (linear scale) of the first fifteen string resonances in open E2 configuration. The $-3 \mathrm{~dB}$ mark is indicated by the dotted line.

string as the guitar under test was tuned slightly flat with a fundamental at $80 \mathrm{~Hz}$. This matches the frequency of the swelling tone observed by the authors during the calibration process of Sec. 3.1.

In particular, sharp resonance peaks at the strings' modal frequencies are shown to exceed the contribution from the soundbox throughout the frequency range. This result provides evidence that instabilities are susceptible to first occur from the string's modes and not from the soundbox, which justifies prioritizing the string instability aspect of the problem over that of the soundbox. In particular, the shape of the magnitude response, exhibiting sharp deviations from the mean, suggests FS as a viable solution to string instability. A detailed view of the resonance peaks is given for the first 15 harmonics in Fig. 6. As pictured, the majority of resonance are narrow with a bandwidth of $1 \mathrm{~Hz}$ at most. For these resonances, a frequency shift of corresponding value would suffice to offset any feedback away from the resonance and prevent instability. However, a relatively wide resonance can also be seen in the figure. This requires opting for a higher frequency offset value to maximize the added gain margin. We limit our choice to an offset of $3 \mathrm{~Hz}$ so as to prevent the occurrence of a vibrato effect as discussed in the introduction.

\subsection{Plucking Experiments without and with FS}

The A-weighted SPL delivered by the augmented guitar setup following calibrated plucks of the low E string is reported for different values of the loop gain in Fig. 7(a). Each curve represents an average of three measurements. In this figure, loop gains are given relative to a reference level, i.e., $0 \mathrm{~dB}$, at which the string's vibration started building up and quickly reached saturation as a result of rattling against the frets. This saturation is apparent in the upper-most SPL curve of the graph. The SPL curves are also reported for lower loop gain levels. In particular, the SPL is shown to decay quickly, when the feedback loop is practically

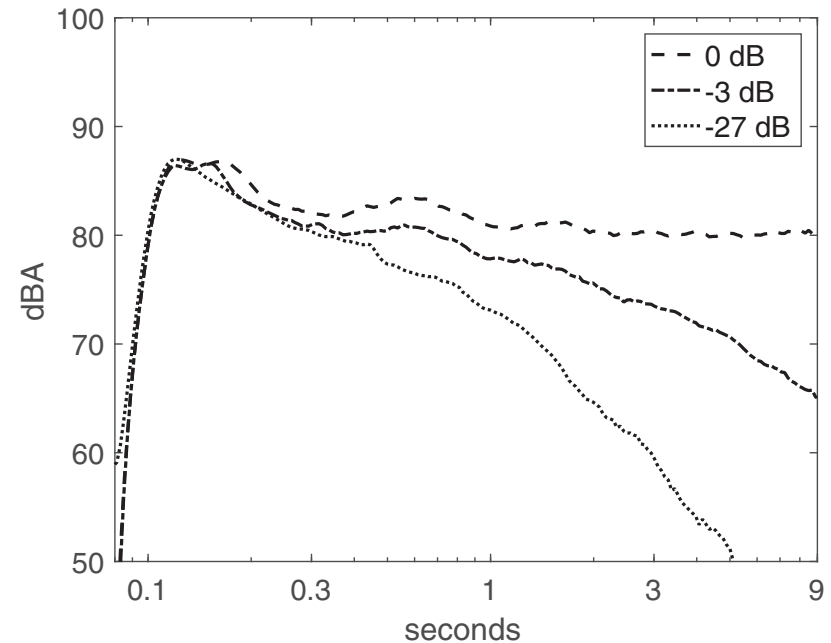

(a)

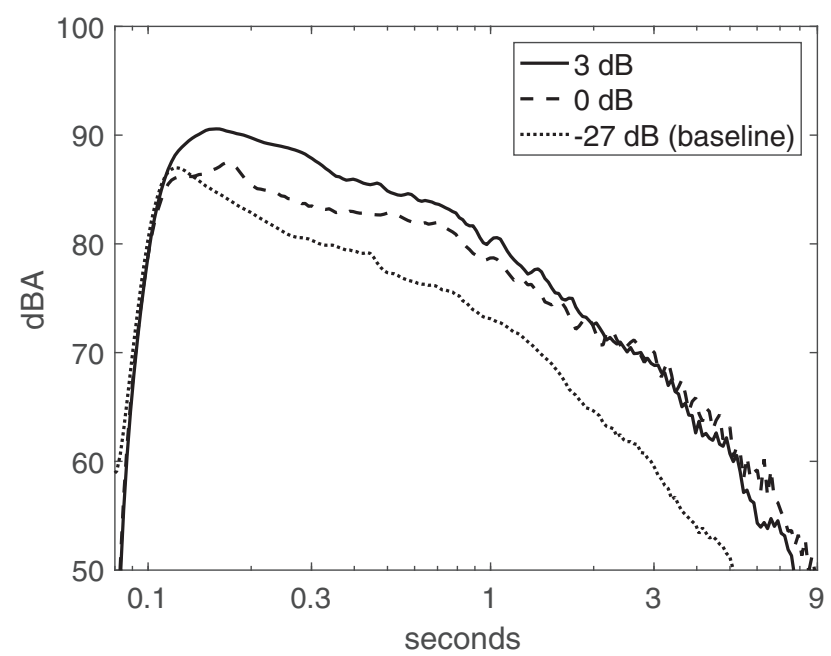

(b)

Fig. 7. Measured average A-weighted SPL of a low E tone played on the augmented guitar, as a function of time for several loop gain setups. Loop gain levels are given relative to that of unstable baseline setup $(0 \mathrm{~dB})$. (a) Baseline without FS and (b) with FS using a $3-\mathrm{Hz}$ frequency shift. The low-gain $(-27 \mathrm{~dB})$ baseline measurement is reproduced in (b) for comparison.

open with a loop gain of $-27 \mathrm{~dB}$. A prolonged sustain was observed for an intermediate loop gain level $-3 \mathrm{~dB}$ under the observed unstable level.

As reported by Fig. 7(b), application of an upwards frequency shift of $3 \mathrm{~Hz}$ mitigates the active sustain phenomenon. It can be seen that the SPL curve for loop gains of both $0 \mathrm{~dB}$ and $3 \mathrm{~dB}$ now decay at a rate comparable to the unmodified augmented guitar setup with lowest loop gain, i.e., of $-27 \mathrm{~dB}$, see dotted curve in Fig. 7(b). In other words, when FS is applied, the additional gain now provides an increased SPL but practically no change in the sustain. FS thus provides an additional gain margin before instability, which improves the usability of the augmented guitar setup. However, this improvement comes at the expense of an audible amplitude modulation, which is discussed in the following section. 


\subsection{Qualitative Evaluation}

A qualitative evaluation of the proposed method is provided by recorded performances on the augmented guitar with and without FS. Specifically, four recordings of the first four chords from the song title Where Is My Mind [29] are provided in a companion web page ${ }^{1}$. The first recording was conducted with the loop gain set to $-3 \mathrm{~dB}$ below the above-mentioned reference level. At that level, the augmented guitar sound exhibits prolonged ringing of the string but without building up. The second recording was conducted with the loop gain set to the reference level $(0 \mathrm{~dB})$. At that level, the guitar sound builds up and saturates at the end of the chord progression. This effect is mitigated in the third recorded example, which was carried out at the same loop gain level $(0 \mathrm{~dB})$ but with FS introduced in the forward path. The fourth and last recorded example shows that stability is preserved for even an increased loop gain level $(3 \mathrm{~dB})$. These results are coherent with those of Fig. 7.

As can be heard from the third and fourth recordings, an amplitude modulation artifact results from use of FS. This artifact is not negligible when evaluated against the natural sound of the guitar. However, the amplitude modulation can be acceptable in the musical usage of the augmented guitar where other audio effects are also introduced in the forward path.

\section{CONCLUSION}

In this article we studied the feedback problem occurring when an amplified guitar pickup signal drives an actuator mounted on the back plate of the guitar. The measurements of the system's open loop response were interpreted in light of the Nyquist stability criterion. Under the reported measurement conditions, instability occurred at modes of the open E2 string for loop gain levels $3 \mathrm{~dB}$ lower than that required for instabilities at the soundbox modes.

Frequency shifting was applied to address the string instability problem and was shown to improve the loop gain margin by at least $3 \mathrm{~dB}$, at the expense of an amplitude modulation effect. This artifact was found to be significant, but potentially acceptable, especially within musical context where audio effects are used to enriching the guitar's tone.

\section{ACKNOWLEDGMENT}

This work is related to the project Active AcousticsSmart Audio Objects at the Interface of the Material and the Digital and was financed in part by the Academy of Finland (project no. 305309 and subproject no. 13305309 at Aalto University). This research was also part of the activities of the "Nordic Sound and Music Computing NetworkNordicSMC," NordForsk project number 86892.

\footnotetext{
${ }^{1} \mathrm{http} / / /$ research.spa.aalto.fi/publications/papers/jaes-2018freqShift/
}

\section{REFERENCES}

[1] T. van Waterschoot and M. Moonen, "Fifty Years of Acoustic Feedback Control: State of the Art and Future Challenges," Proc. IEEE, vol. 99, no. 2, pp. 288-327 (2011 Feb.), doi:https://doi.org/10.1109/JPROC.2010.2090998.

[2] P. U. Svensson, "Computer Simulations of Periodically Time-Varying Filters for Acoustic Feedback Control," J. Audio Eng. Soc., vol. 43, pp. 667-677 (1995 Sep.).

[3] J. L. Nielsen and U. P. Svensson, "Performance of Some Linear Time-Varying Systems in Control of Acoustic Feedback," J. Acoust. Soc. Amer., vol. 106, no. 1, pp. 240 254 (1999 Jul.), doi:https://doi.org/10.1121/1.427053.

[4] M. R. Schroeder, "Improvement of Acoustic Feedback Stability in Public Address Systems," J. Acoust. Soc. Amer, vol. 31, no. 6, pp. 851-852 (1959 Apr.).

[5] M. R. Schroeder, "Stop Feedback in Public Address Systems," Radio Electron., vol. 31, pp. 40-42 (1960 Feb.).

[6] M. R. Schroeder, "Improvement of Feedback Stability of Public Address Systems by Frequency Shifting," $J$. Audio Eng. Soc., vol. 10, no. 2, pp. 108-109 (1962 Apr.).

[7] M. R. Schroeder, "Improvement of AcousticFeedback Stability by Frequency Shifting," J. Acoust. Soc. Amer., vol. 36, no. 9, pp. 1718-1724 (1964 Sep.), doi:https://doi.org/10.1121/1.1919270.

[8] M. R. Schroeder, "Die statistischen Parameter der Frequenzkurven von großen Räumen, "Acustica, vol. 4, no. 5, pp. 594-600 (1954 Jan.).

[9] M. R. Schroeder, "Statistical Parameters of the Frequency Response Curves of Large Rooms," J. Audio Eng. Soc., vol. 35, pp. 299-306 (1987 May).

[10] M. A. Poletti, "The Stability of Single and Multichannel Sound Systems," Acta Acustica united with Acustica, vol. 86, no. 1, pp. 163-178 (2000 Jan.).

[11] C. Zheng, C. Hofmann, X. Li, and W. Kellermann, "Analysis of Additional Stable Gain by Frequency Shifting for Acoustic Feedback Suppression Using Statistical Room Acoustics," IEEE Signal Processing Letters, vol. 23, no. 1, pp. 159-163 (2016 Jan.), doi:https://doi.org/10.1109/LSP.2015.2507205.

[12] E. J. Berdahl, "Applications of Feedback Control to Musical Instrument Design," Ph.D. thesis, Stanford University, Stanford, CA (2009 Dec.).

[13] E. Berdahl, J. O. Smith, and G. Niemeyer, "Feedback Control of Acoustic Musical Instruments: Collocated Control Using Physical Analogs," J. Acoust. Soc. Amer., vol. 131, no. 1(2), pp. 963-973 (2012 Jan.), doi:https://doi.org/10.1121/1.3651091.

[14] O. Lähdeoja, "Active Acoustic Instruments for Electronic Chamber Music," Proceedings of the International Conference on New Interfaces for Musical Expression, vol. 16, pp. 132-136 (Brisbane, Australia) (2016 July).

[15] I. Perry, "Sound Radiation Measurements on Guitars and Other Stringed Musical Instruments," Ph.D. thesis, Cardiff University, Cardiff, UK (2014 Sep.).

[16] S. Griffin, S. A. Lane, and R. L. Clark, "The Application of Smart Structures Toward Feedback Suppression in Amplified Acoustic Guitars," J. Acoust. Soc. 
Amer., vol. 113, no. 6, pp. 3188-3196 (2003 Jun.), doi:https://doi.org/10.1121/1.1562649.

[17] S. Benacchio, B. Chomette, A. Mamou-Mani, and V. Finel, "Mode Tuning of a Simplified String Instrument Using Time-Dimensionless State-Derivative Control," J. Sound and Vibration, vol. 334, pp. 178-189 (2015 Jan.), doi:https://doi.org/10.1016/j.jsv.2014.09.003.

[18] C. Gough, "Modelling and Synthesis of Violin Vibrato Tones," Forum Acusticum, pp. 721-726 (2005 Sep.).

[19] M. Mellody and G. H. Wakefield, "The TimeFrequency Characteristics of Violin Vibrato: Modal Distribution Analysis and Synthesis," J. Acoust. Soc. Amer., vol. 107, no. 1, pp. 598-611 (2000 Jan.), doi:https://doi.org/10.1121/1.428326.

[20] L. C. Westphal, "Nyquist Stability Theory," in Handbook of Control Systems Engineering, chap. 15, pp. 343-364 (Springer, 2001), doi:https://doi.org/10.1007/ 978-1-4615-1533-3_15.

[21] H. Nyquist, "Regeneration Theory," Bell Labs Tech. J., vol. 11, no. 1, pp. 126-147 (1932 Jan.), doi:https://doi.org/10.1002/j.1538-7305.1932.tb02344.x.

[22] M. Puckette, The Theory and Technique of Electronic Music (World Scientific Publishing Company, 2007), doi:https://doi.org/10.1142/6277.
[23] U. Zölzer, DAFX: Digital Audio Effects, 2nd ed. (John Wiley \& Sons, 2011), doi:https:// doi.org/10.1002/9781119991298.

[24] S. K. Mitra and J. F. Kaiser, Handbook for Digital Signal Processing (John Wiley \& Sons, Inc., 1993).

[25] A. Farina, "Simultaneous Measurement of Impulse Response and Distortion with a Swept-Sine Technique," presented at the 108th Convention of the Audio Engineering Society (2000 Feb.), convention paper 5093.

[26] A. Novak, L. Simon, F. Kadlec, and P. Lotton, "Nonlinear System Identification Using Exponential Swept-Sine Signal," IEEE Trans. Instr. Meas., vol. 59, no. 8, pp. 2220-2229 (2010 Aug.), doi:https://doi.org/ 10.1109/TIM.2009.2031836.

[27] A. Farina, "Advancements in Impulse Response Measurements by Sine Sweeps," presented at the 122nd Convention of the Audio Engineering Society (2007 May), convention paper 7121 .

[28] M. Mustonen, D. Kartofelev, A. Stulov, and V. Välimäki, "Experimental Verification of Pickup Nonlinearity," Proceedings International Symposium on Musical Acoustics (ISMA 2014), Le Mans, France, vol. 1, pp. 651656 (2014 July).

[29] C. Thompson, "Where Is My Mind?" [Recorded by The Pixies], On Surfer Rosa [CD], Elektra, 2003 (1988). 


\section{THE AUTHORS}

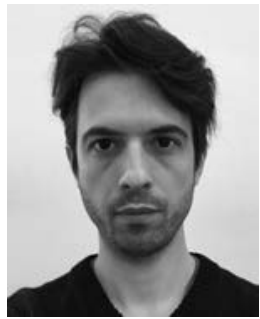

Etienne Thuillier

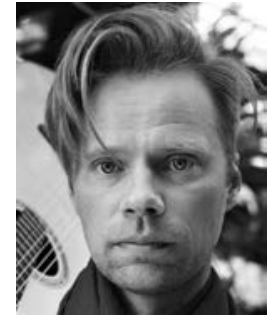

Otso Lähdeoja

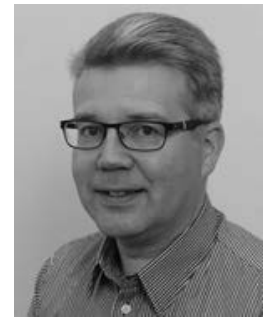

Vesa Välimäki
Etienne Thuillier is a Ph.D. student from Aalto University (Espoo, Finland). His research lies at the intersection of psychoacoustics and machine learning with a focus on providing engaging human-machine interfaces. Originally a telecommunications engineer, Etienne Thuillier started his career as a patent consultant before re-converting to technical practice through a startup creation experience and master's studies in audio signal processing and acoustics at Aalto University.

Otso Lähdeoja is a music technology researcher, composer and guitarist, and postdoctoral researcher at the University of the Arts Helsinki (Finland). He holds a Ph.D. from University of Paris VIII (France), and has led research projects on instrument augmentation, structureborne sound in music and intermedia creation, as well as on active acoustic instruments. He is currently the Principal Investigator of the Academy of Finland key project entitled "Active Acoustics - Smart Audio Objects at the Interface of the Material and the Digital."

Vesa Välimäki is a Full Professor of audio signal processing and Vice Dean of Research at Aalto University, Espoo, Finland. He received his M.Sc. in Technology and
Doctor of Science in Technology degrees, both in electrical engineering, from the Helsinki University of Technology (TKK) in 1992 and 1995, respectively. His doctoral dissertation dealt with fractional delay filters and physical modeling of musical instruments. In 1996 he was a Postdoctoral Research Fellow at the University of Westminster, London, UK. In 2001-2002 he was Professor of signal processing at the Pori School of Technology and Economics, Tampere University of Technology, Pori, Finland. In 2002, he was appointed Professor of audio signal processing at TKK. During the academic year 2008-2009 he was on sabbatical as a Visiting Scholar at the Center for Computer Research in Music and Acoustics (CCRMA), Stanford University, Stanford, CA. His research interests include audio effects processing, digital filter design, sound synthesis, and acoustics of musical instruments. Prof. Välimäki is a Fellow of the IEEE, a Fellow of the AES, and a Life Member of the Acoustical Society of Finland. Since 2015, he has been a Senior Area Editor of the IEEE/ACM Transactions on Audio, Speech and Language Processing. He was a Guest Editor of the Music Signal Processing special issue of the IEEE Signal Processing Magazine in 2019. He was the General Chair of the 14th Sound and Music Computing Conference SMC-17 in 2017. 